\title{
Small-amplitude trochoidal oscillations in Typhoons Rammasun (2014) and Lekima (2019)
}

\author{
Yecheng Feng ${ }^{1,3}$ and Liguang $\mathrm{Wu}^{2,4, *}$ \\ ${ }^{1}$ Key Laboratory of Meteorological Disaster of Ministry of Education, Nanjing University of Information Science and Technology, \\ Nanjing, China \\ ${ }^{2}$ Department of Atmospheric and Oceanic Sciences and Institute of Atmospheric Sciences, Fudan University, Shanghai, China \\ ${ }^{3}$ State Key Laboratory of Severe Weather, Chinese Academy of Meteorological Sciences, Beijing, China \\ ${ }^{4}$ Innovation Center of Ocean and Atmosphere System, Zhuhai Fudan Innovation Research Institute, Zhuhai, China
}

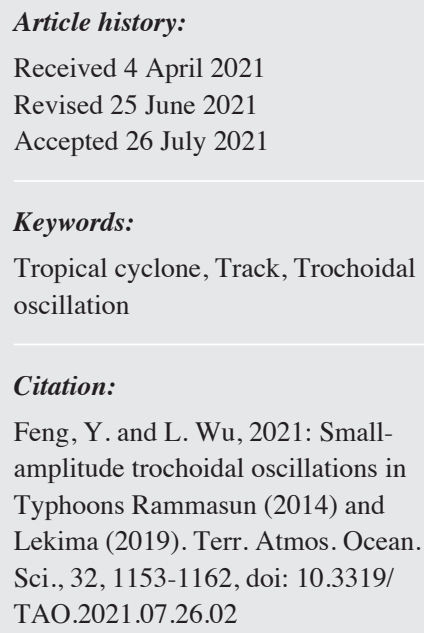
amplitude trochoidal oscillations in Typhoons Rammasun (2014) and Lekima (2019). Terr. Atmos. Ocean. Sci., 32, 1153-1162, doi: 10.3319/ TAO.2021.07.26.02

\begin{abstract}
Tropical cyclones (TCs) tend to oscillate around a mean path and the trochoidal oscillation has been documented based on radar, satellite and aircraft observational data and in numerical simulations. Currently, no consensus has been reached on the mechanisms responsible for the trochoidal oscillation in TC motion although a few theories have been proposed since 1950. In this study, the small- amplitude track oscillations of Super Typhoons Rammasun (2014) and Lekima (2019) are examined based on the 6-minute radar refelctivity and the detected oscillation in Rammasun is verified through numerical simulation. The trochoidal oscillation in the tracks of Super Typhoons Rammasun and Lekima can be retrieved with the 6-minute radar data. The detected track oscillation always exhibits cyclonic rotations around a mean track, with the amplitude of a few kilometers and the period of a few hours. The numerical simulation of Typhoon Rammasun indicates that the small-amplitude track oscillation can be simulated in terms of the period and amplitude. Based on two different center-detecting methods, it is shown that the simulated track oscillation represents the wobble of the whole inner-core circulation. Since the small-scale oscillations are closely associated with the dynamics of the TC inner-core circulation, this study suggests that the trochoidal oscillation in TC tracks may provide important information for understanding the intensity and structure changes of TCs.
\end{abstract}

\section{INTRODUCTION}

Tropical cyclones (TCs) tend to meander or oscillate around a mean track. The track oscillation covers a wide range of periods ranging from less than one hour to a few days (Holland and Lander 1993). A typical form of the track oscillation is short-period, small-amplitude wobbles around otherwise relatively smooth track, which has been documented based on radar, satellite and aircraft data (e.g., Horn 1951; Jordan and Stowell 1955; Lawrence and Mayfield 1977; Muramatsu 1986; Hong and Chang 2005; Marks et al. 2008) and found in numerical simulations (e.g., Jones 1977; Abe 1987; Nolan et al. 2001; Wu and Chen 2016; Yang et al. 2020). Some studies also found that the track oscillation exhibits a cyclonic rotation around the mean track (Abe 1987;

\footnotetext{
* Corresponding author

E-mail:liguangwu@fudan.edu.cn
}

Hong and Chang 2005; Marks et al. 2008; Wu and Chen 2016; Yang et al. 2020). Recent numerical studies suggested that the trochoidal oscillation was the wobble of the whole inner-core circulation (Wu and Chen 2016; Menelaou et al. 2018; Yang et al. 2020), rather than the displacement of the inner eyewall in TCs with concentric eyewalls (Jordan 1966; Muramatsu 1986; Hong and Chang 2005; Oda et al. 2006) or the minimum pressure or streamfunction center due to the inner-core vorticity mixing (Nolan et al. 2001).

Many previous studies have been conducted to understand the track oscillation since the pioneering work of Yeh (1950). He found the trochoidal motion when a TC was embedded in the easterly environmental flow. The amplitude and period of the trochoidal oscillation depended on the environmental flow and the size and intensity of the storm. Since then, various theories have been proposed to understand the trochoidal motion of TCs. Kuo (1969) and 
Jones (1977) attributed the trochoidal oscillation around a TC mean track to frictional drag force, while Khandekar and Rao (1971) suggested that the short-period track oscillation resulted from the interaction of a sloping vortex between the upper-level and lower-level circulations. Reasor and Montgomery (2001) argued that the trochoidal motion is linked to the vertical shear of the environment flow. The resulting potential vorticity (PV) anomalies in the tropopause and the surface tend to co-rotate. In addition, the wave-number-one instability, which lead to substantial inner-core vorticity redistribution and mixing, may be responsible for the trochoidal motion of the TC-like barotropic vortex (Nolan et al. 2001). Nolan et al. (2001) suggested that the trochoidal oscillation is the displacement of the minimum pressure or streamfunction center other than the whole inner-core circulation. It can be seen that no agreement has been reached about the mechanisms for the trochoidal oscillation in TC motion.

Some studies argued that the trochoidal motion are due to the asymmetric concentric eyewalls (Jordan 1966; Muramatsu 1986; Hong and Chang 2005; Oda et al. 2006). Hong and Chang (2005) pointed out that the inner eye tended to circulate due to the steering of the rotational flow in the moat between the inner and outer eyewalls, resulting in the oscillation around the mean track. Oda et al. (2006) suggested that the concentric eyewall structure can be treated as a double vortex composed of the inner vortex and the outer vortex ring and the trochoidal motion can result from the interaction between two parts of an asymmetric double vortex. Recently, Menelaou et al. (2018) indicated that instability induced by the coupling of two baroclinic vortex Rossby waves across the moat region is the triggering mechanism for the oscillating wobbles in the simulated TC with concentric eyewalls. These studies tend to attribute the trochoidal oscillation of TC tracks to the steering associated with the outer eyewall.

Wu and Chen (2016) and Yang et al. (2020) examined the track oscillation of the simulated TCs with the amplitude comparable to the eye size and periods of several hours and found that the track oscillation is the cyclonic rotation of the TC relative to the mean track, in agreement with previous observational and numerical studies (Lawrence and Mayfield 1977; Muramatsu 1986; Willoughby 1988; Nolan et al. 2001; Itano et al. 2002). While most previous studies linked the trochoidal motion to the steering effect, $\mathrm{Wu}$ and Chen (2016) indicated that the trochoidal motion with amplitudes smaller than the eye radius and periods of several hours cannot be accounted for by the steering effect. Using four different methods for detecting the TC center position in the numerical experiment, Yang et al. (2020) found the similar track oscillations, implying that the trochoidal oscillation was the wobble of the whole inner-core circulation.

As the first and fifth strongest landfall typhoon in the Chinese mainland, Super Typhoons Rammasun (2014) and
Lekima (2019) made landfall in South China and East China with a single eyewall and concentric eyewalls, respectively. Due to their strong convection structure, the eyes of Rammasun and Lekima had been captured by the coastal S-band Doppler radar and the tracks were then accurately detected based on the radar reflectivity. In this study, using the 6-minute radar data, small-amplitude trochoidal oscillations of the tracks of Super Typhoons Rammasun (2014) and Lekima (2019) with different tracks and structures are documented during their landfall. Moreover, the track oscillation of Super Typhoons Rammasun (2014) is simulated and compared with the observation in terms of the period and amplitude.

\section{DATA AND METHODS}

In this study, the small-amplitude oscillation of the TC track is defined as the wobbles relative to the 3-h and 6-h mean tracks. The track oscillations are based on the radar data from two coastal S-band Doppler radars at Haikou, Hainan Province and Wenzhou, Zhejiang Province. Due to the limitation of the radar detection range, our analysis period starts when the TC eye was fully captured by the radar and ends when the storms made landfall. The range and azimuthal resolutions of the radar data are $1 \mathrm{~km}$ and $1^{\circ}$ at 6-minute intervals, respectively. For comparison, we also use the real-time monitoring of the 1-h track and intensity from China Meteorological Administration (CMA) and the best track dataset of the Shanghai Typhoon Institute (STI) of the CMA at 6- intervals (Ying et al. 2014; Lu et al. 2021).

The 6-minute TC center was detected by assuming that the eyewall is a circle and the geometric center of the circle is defined as the TC center (Chang et al. 2009). We use the radar reflectivity at $0.5^{\circ}$ elevation to detect the TC center and assume that the impact of the vertical tilt of the radar data slice with the increasing range on the center position is negligible. The radar data are interpolated to the Cartesian coordinates at the grid spacing of $1 \mathrm{~km}$. The radius and center of the circle are adjusted until the minimum mean reflectivity over the circle is reached. The TC center is the geometric center of the circle with the minimum mean reflectivity. A 1-h running average is applied to the radar-based centers to reduce the noise in the detected track. Figure 1 shows the detected centers of Typhoon Rammasun at 0534 UTC 18 July 2014 and Typhoon Lekima at 1239 UTC 9 August 2019. The horizontal axis and the vertical axis indicate the zonal and meridional distance to the location of the radar station. We can see that the detected centers are visually reasonable.

Figure 2 further shows the comparisons between the CMA real-time monitoring and the radar-based track. Note that the latter does not exactly correspond with the real-time monitoring in time, while the detected tracks are generally consistent with the CMA real-time monitoring. The main reason for the difference between the real-time monitoring 

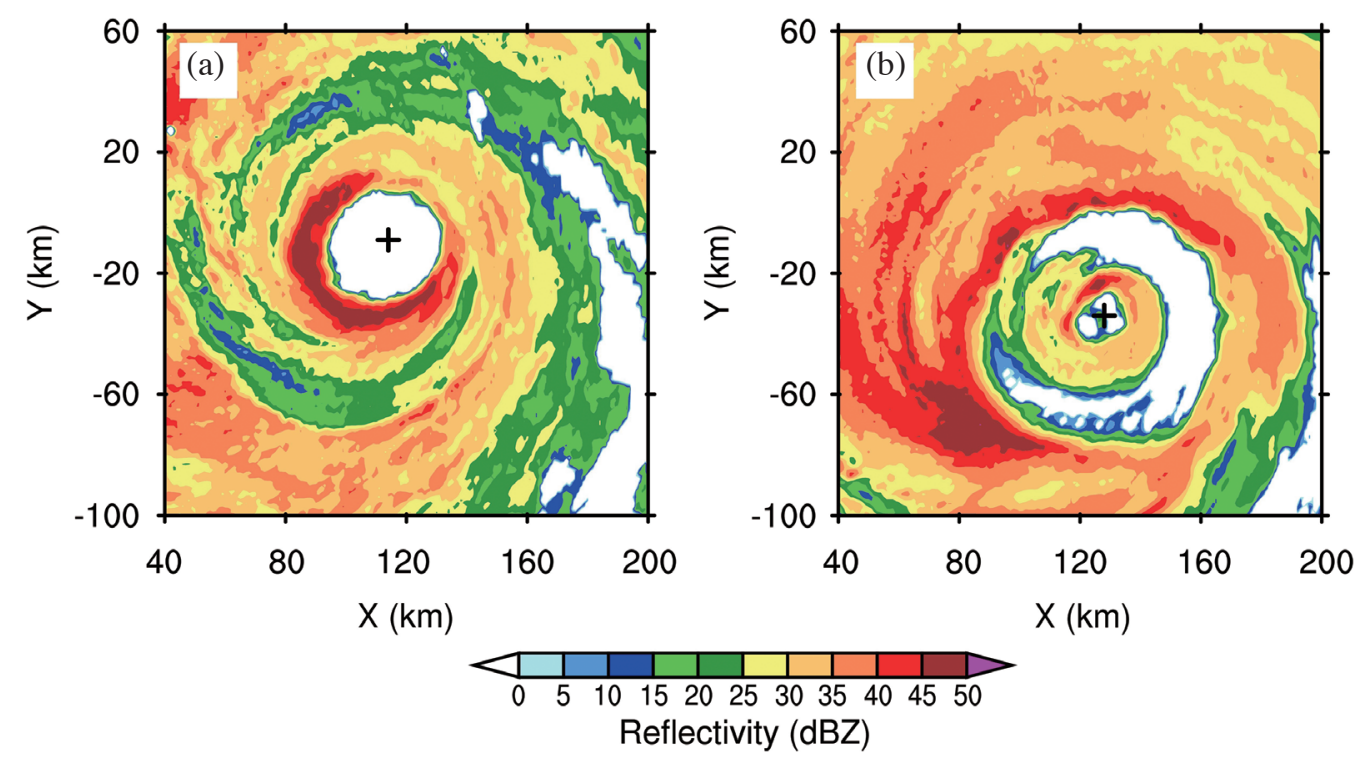

Fig. 1. The S-band Doppler radar reflectivity (dBZ) of (a) Typhoon Rammasun at 0534 UTC 18 July 2014 and (b) Typhoon Lekima at 1239 UTC 9 August 2019, with the plus signs indicating the tropical cyclone center. The $\mathrm{x}$ and y coordinates indicates the zonal and meridional distance to the location of the radar station, respectively.
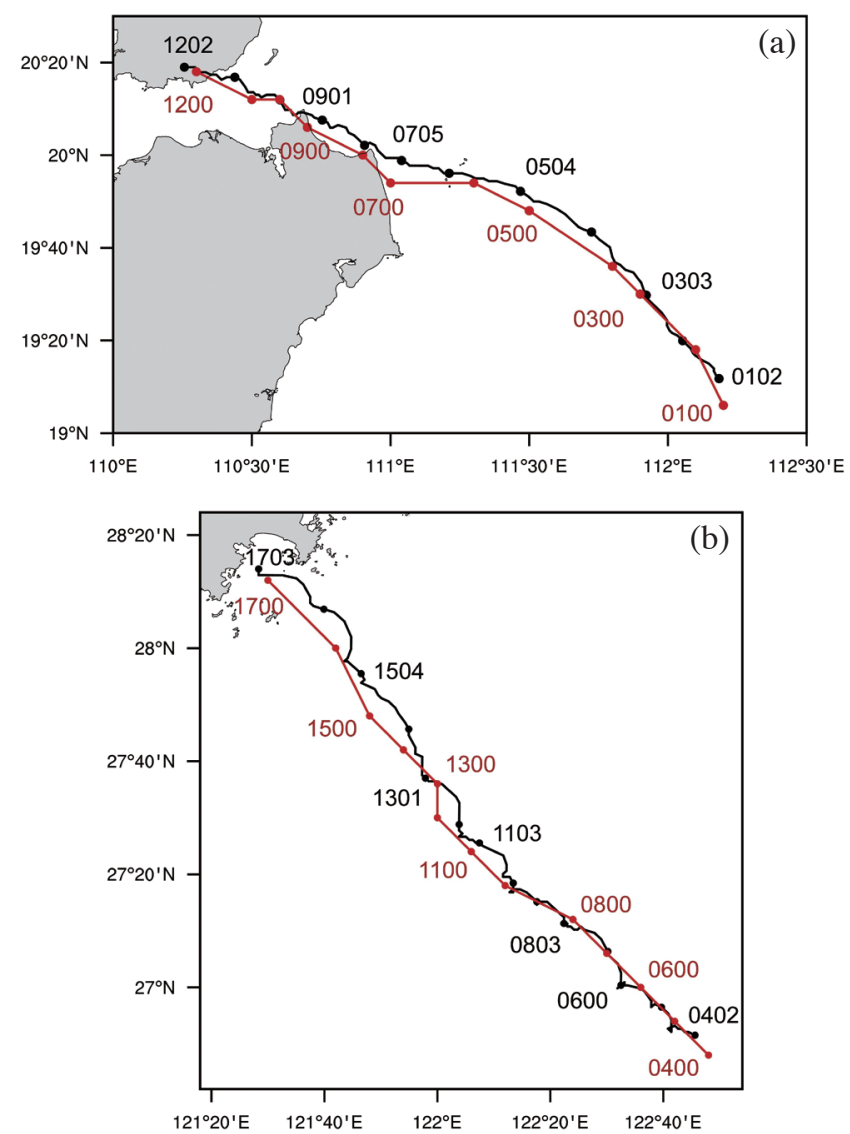

Fig. 2. The 1-h track issued by CMA (red) and the 6-minute track detected with radar reflectivity (black) for (a) Typhoon Rammasun on 18 July 2014 and (b) Typhoon Lekima on 9 August 2019. The UTC time is indicated by the form of HHMM. 
and the radar-based track is that the center position in the CMA real-time monitoring is accurate only to one decimal place in latitude and longitude. That is, its error is about $10 \mathrm{~km}$. In addition, the CMA real-time monitoring is also based on the data from satellite and station observation (Feng et al. 2021). Our following discussion will show that the CMA real-time monitoring cannot capture the smallamplitude trochoidal oscillation of the tracks of Super Typhoons Rammasun (2014) and Lekima (2019) since the oscillation amplitude is generally less than $10 \mathrm{~km}$.

As mentioned in section 1, Yang et al. (2020) examined the performance of four center-detecting methods in the simulated Hurricane Wilma (2005) with the finest horizontal grid spacing of $1 \mathrm{~km}$. The selected four methods can be divided into two categories: one is called weighted grid point (WGP), including the pressure centroid center (PCC) and the potential vorticity (PV) centroid center (PVC), and the other category includes the maximum tangential wind center (MTC) and the minimum pressure variance center (MVC). While the azimuthal variance of pressure in the inner core is minimized with the MVC, the azimuthal mean tangential wind is maximized with the MTC. In other words, the centers detected with the MVC and MTC represent the center of the inner-core circulation, rather than the minimum pressure or streamfunction center.

\section{THE OBSERVED SMALL-AMPLITUDE TRACK OSCILLATIONS}

\subsection{Overview of Typhoons Rammasun and Lekima}

Typhoon Rammasun (2014) formed to the east of the Philippines on 12 July, intensifying into the super typhoon strength in the South China Sea on 17 July. After 36 hours of rapid intensification, it reached its peak intensity at 0600 UTC 18 July. In the real-time monitoring released by China Meteorological Administration (CMA), Joint Typhoon Warning Center (JTWC), and Japan Meteorological Agency (JMA), the central pressure at its peak intensity was 910 , 922 , and $935 \mathrm{hPa}$, respectively (Cai and Xu 2016). Cai and $\mathrm{Xu}$ (2016) argued that the central pressure of Rammasun should be lower than $888 \mathrm{hPa}$ with the maximum wind speed of $70-76 \mathrm{~m} \mathrm{~s}^{-1}$. Rammasun made landfall over Hainan Island at 0730 UTC 18 July 2014, and made a second landfall in Guangdong Province just four hours later (Fig. 2a). It was the strongest landfall typhoon ever recorded in the Chinese mainland. Rammasun caused at least 30 deaths and over 26.5 billion RMB direct economic losses in China.

As the fifth strongest landfall typhoon in the Chinese mainland, Super Typhoon Lekima (2019), similar to Rammasun, also formed to the east of the Philippines on $4 \mathrm{Au}-$ gust. It moved to the East China Sea in the following days and experienced the rapid intensification from 1200 UTC 6 to 0900 UTC 8 August. As shown in Fig. 2b, Lekima made landfall in Wenling City, Zhejiang Province at 1745 UTC 9
August, maintaining the concentric eyewalls structure, with the two-minute sustained wind of $52 \mathrm{~m} \mathrm{~s}^{-1}$ and the central pressure of $930 \mathrm{hPa}$. Lekima inflicted 57 deaths and 53.72 billion RMB direct economic losses in China.

As shown in Fig. 1a, Typhoon Rammasun exhibited the significant asymmetry in the radar reflectivity at 0534 UTC 18 July 2014, when it nearly finished the 36-h rapid intensification process and reached its peak intensity. The strongest convection occurred in the western and southern semicircle of the eyewall. Qiu et al. (2020) indicated that the asymmetry structure of the eyewall was closely associated with the vertical wind shear of about $10 \mathrm{~m} \mathrm{~s}^{-1}$ in the environmental wind. The rapid intensification process of Rammasun experienced the rapid intensification in a sheared environment. As shown in Fig. 1b, Typhoon Lekima displayed the concentric eyewalls, which persisted for at least 15 hours until the typhoon made landfall in eastern China (Bao et al. 2020; Dai et al. 2021). Due to the presence of the concentric eyewalls, the eye of Lekima is smaller than that of Rammasun.

\subsection{Small-Amplitude Track Oscillations}

As shown in Fig. 2, there are trochoidal oscillations in the radar-based tracks of Typhoons Rammasun and Lekima. In order to clearly demonstrate the trochoidal oscillations, we calculated the 3-h and 6-h running means of the TC center position and removed the mean track from the original tracks. The selection of the average periods is due to the effective range of the radar observation. There are 11 and 13 hours of 6-minute center positions for Rammasun and Lekima, respectively. In Wu and Chen (2016) and Yang et al. (2020), an average period of 9 hours was selected. In the following discussion, we will show that the relatively short average period leads to the smaller amplitude of the trochoidal oscillation.

Figures $3 \mathrm{a}$ and $\mathrm{c}$ show the time series of the zonal and meridional radar-based track oscillations relative to the 3-h mean tracks of Rammasun and Lekima. We can see that the meridional oscillations always follow the zonal oscillations for the two typhoons, suggesting a fixed pattern of the rotation of the TC center relative to the mean track. The oscillation period of Rammasun was $\sim 4$ hours, which can be divided into two stages: from 0233 UTC to 0700 UTC 18 July with an amplitude of $\sim 3 \mathrm{~km}$, and from 0700 UTC to 1032 UTC 18 July with an amplitude of $\sim 1 \mathrm{~km}$. For Typhoon Lekima, the oscillation period is $\sim 2$ hours with a relatively stable amplitude of $\sim 2 \mathrm{~km}$. We can see that the tracks of the two typhoons show small-amplitude oscillations with periods of a few hours.

To further understand the trochoidal oscillation in the tracks of the two typhoons, we plot the trajectory of the track oscillation relative to the mean track (Fig. 4). In agreement with previous studies (e.g., Wu and Chen 2016; Yang 
(a)

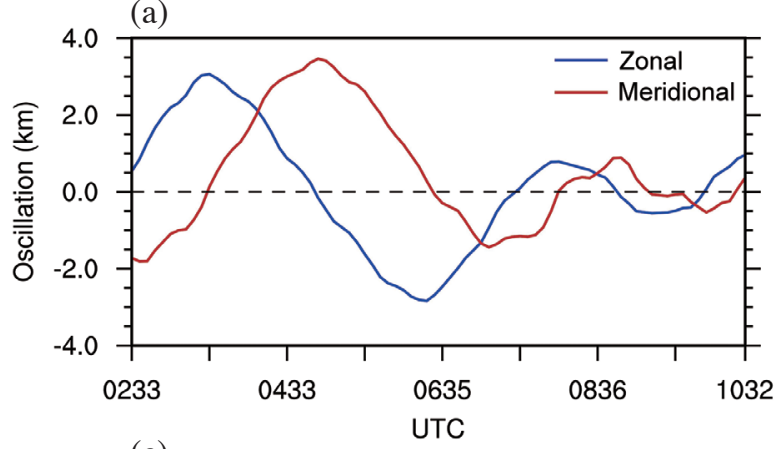

(c)

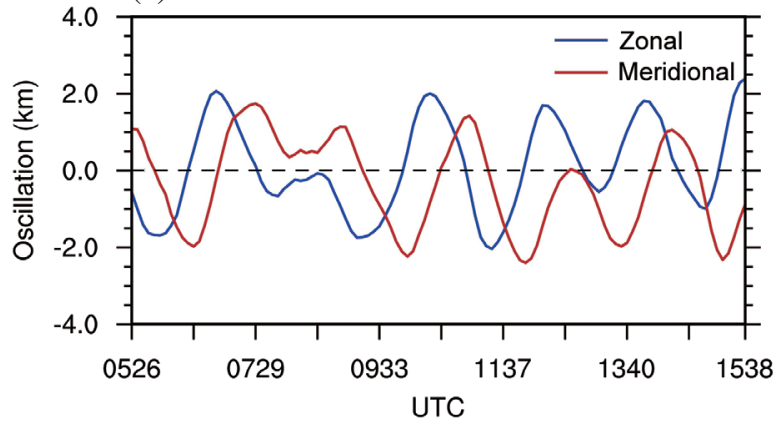

(b)

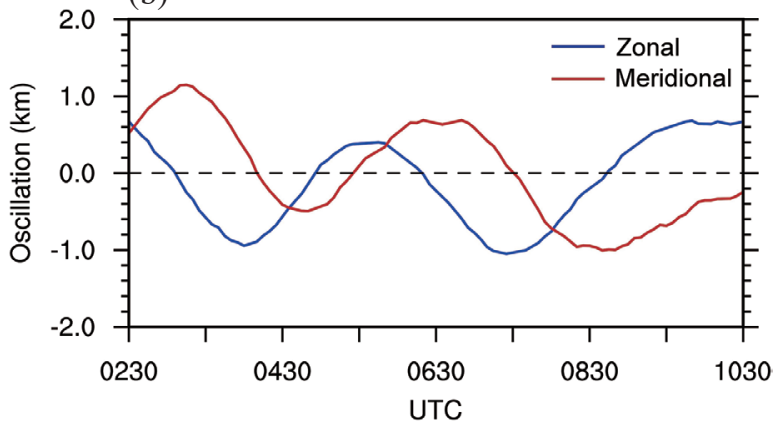

Fig. 3. Time series of the zonal (blue) and meridional (red) oscillations relative to the 3-h mean tracks of the (a) observation and (b) simulation of Typhoon Rammasun on 18 July 2014 and the (c) observation of Typhoon Lekima on 9 August 2019.
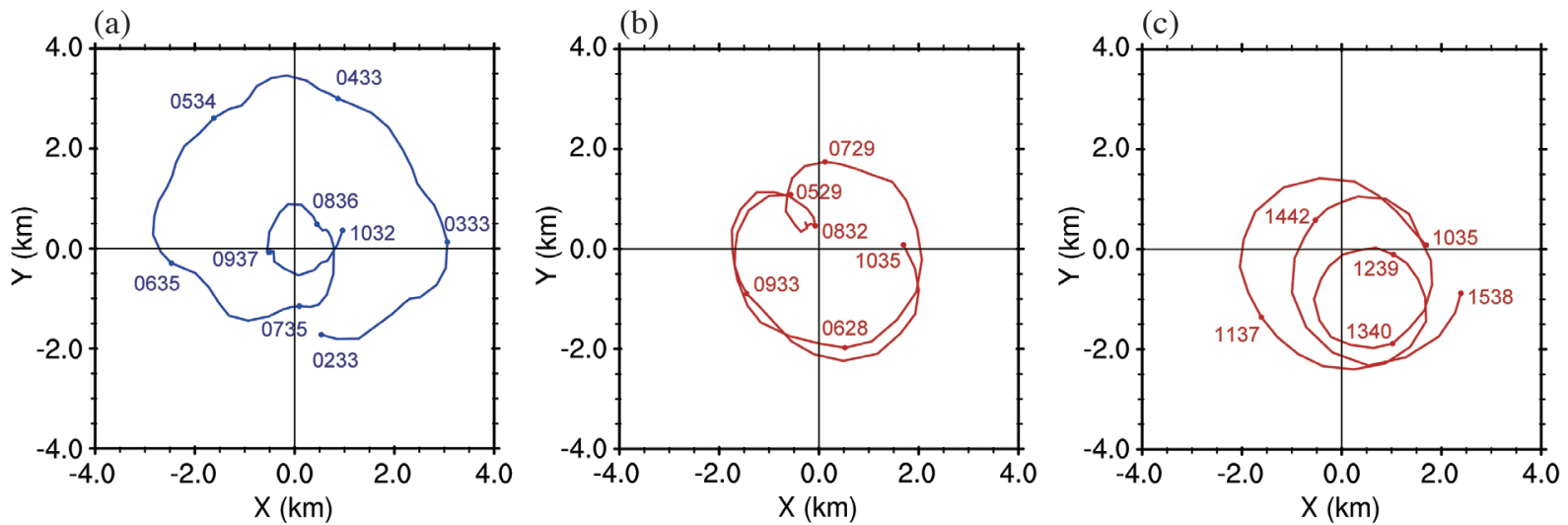

Fig. 4. The track oscillations (km) relative to the 3-h mean tracks of (a) Typhoon Rammasun (blue line) for the period from 0233 UTC to 1032 UTC on 18 July 2014 and (b) Typhoon Lekima for the period from 0526 UTC to 1035 UTC and (c) Typhoon Lekima for the period from 1035 UTC to 1538 UTC on 9 August 2019. The UTC time is indicated by the form of HHMM.

et al. 2020), the TC center always rotates cyclonically relative to the 3-h mean track. For Rammasun, there were two cyclonic circles. The period and amplitude are $\sim 5$ hours and $\sim 3 \mathrm{~km}$ for the larger circle and $\sim 2$ hours and $1 \mathrm{~km}$ for the smaller circle. For Lekima, there are several circles with the amplitude less than $2 \mathrm{~km}$ and the period of $1-2$ hours.

We also examined the track oscillations relative to the 6-h running mean track. Note that the duration was shorter than that relative to the 3 -h running mean due to the limita- tion of the observation period. As shown in Fig. 5, the oscillation with respect to the 6-h running mean track also leads to the cyclonic rotations, but with twice the amplitude of the oscillation relative to the 3 -h running mean. The oscillating pattern of Rammasun was similar to that relative to the 3-h running mean, while the track oscillation of Lekima turned into a larger cyclonic rotation with the two embedded rotations. It is indicated that the cyclonic rotation is a robust feature for the small-amplitude track oscillation. 
(a)

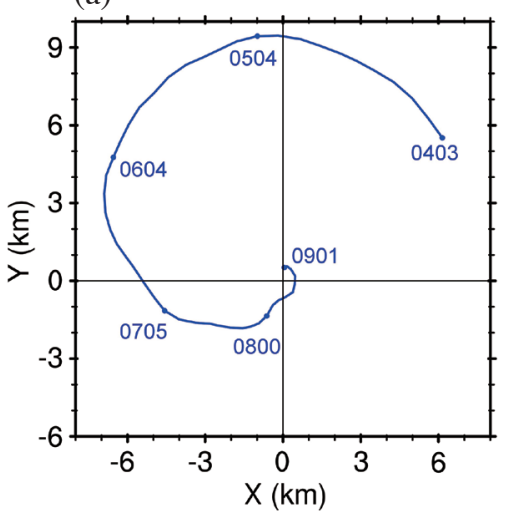

(b)

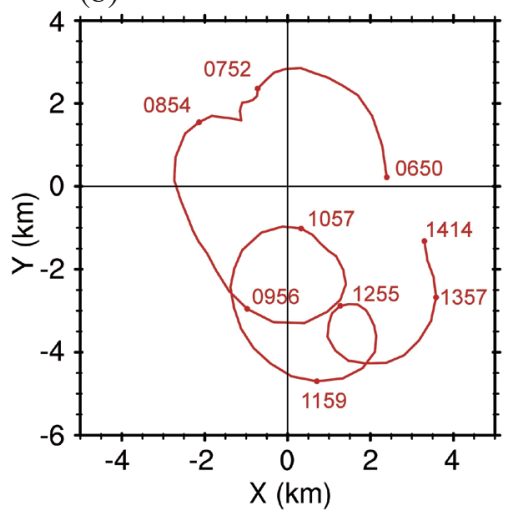

Fig. 5. The track oscillations (km) relative to the 6-h mean tracks of Typhoon Rammasun (blue line) from 0403 UTC to 0901 UTC on 18 July 2014 (a) and Typhoon Lekima (red line) from 0650 UTC to 1414 UTC on 9 August 2019 (b). The UTC time is indicated by the form of HHMM.

\section{SIMULATION OF THE TRACK OSCILLATION OF TYPHOON RAMMASUN (2014)}

\subsection{Experimental Design}

In this study, the numerical simulation of Typhoon Rammasun (2014) was conducted using the WRF model (version 3.2.1) with the outermost domain centered at $19^{\circ} \mathrm{N}$, $116^{\circ} \mathrm{E}$. The initial and boundary conditions are from the National Centers for Environmental Prediction (NCEP) Final (FNL) Operational Global Analysis data. The four two-way interactive domains contain $251 \times 311,271 \times 271,211 \times$ $211,541 \times 541$ grid points, respectively. The corresponding horizontal resolutions are 18,6,2, and 2/3 km ( 667 $\mathrm{m})$. Except the outermost domain, the other three domains are movable to follow the simulated storm. To match the temporal resolution of the observations, the model output during the period from 1200 UTC 16 July to 1200 UTC 18 July was stored at 5-minute intervals.

The Kain-Fritsch cumulus parameterization scheme and the WRF Single-Moment 3-class microphysics scheme (WSM3) are used in the outermost domain (Kain and Fritsch 1993). The WRF Single-Moment 6-class microphysics scheme (WSM6) is used in the nested domains with no cumulus parameterization scheme (Hong and Lim 2006). The other model physics options are the Rapid Radiative Transfer Model (RRTM) longwave radiation scheme (Mlawer et al. 1997) and the Dudhia shortwave radiation scheme (Dudhia 1989). The LES technique is adopted for the sub-kilometer domains (Mirocha et al. 2010), while the Yonsei University scheme is used for planetary boundary layer (PBL) parameterization in the other domains (Noh et al. 2003).

Figure 6 shows the comparisons of the track and intensity from the CMA best track dataset with the simulation during the period 1200 UTC 16 July to 1200 UTC 18 July. The simulated TC generally moves northwestward although the simulated motion is faster than the observation during the period from 0000 UTC 17 July to 0000 UTC
18 July (Fig. 6a). The rapid intensification process was also simulated with the peak intensity of $84 \mathrm{~m} \mathrm{~s}^{-1}$ in the nearsurface maximum wind speed and $897 \mathrm{hPa}$ in the minimum sea level pressure, which are generally consistent with the observation.

\subsection{Simulated Small-Scale Trochoidal Oscillations}

In this section, the simulated TC centers were first detected with the MVC method (Yang et al. 2020). For comparison, the simulated track oscillations were also calculated by removing the 3-h and 6-h running mean tracks. Figures $7 \mathrm{a}$ and $\mathrm{b}$ show two examples of the resulting trajectories of the track oscillation at $3 \mathrm{~km}$ height: one is for the period from 0600 UTC 17 July to 1400 UTC 17 July and the other is for the period from 2200 UTC 17 July to 0600 UTC 18 July. The oscillations relative to the 3-h and 6-h running mean tracks lead to the similar cyclonic rotations. The simulated oscillation amplitudes relative to the 6-h running mean tracks are almost twice of that relative to the 3-h running mean tracks, similar to the observation. The track oscillation relative to the 3-h running mean track contains two types of cyclonic rotations: One is around the mean track and the other is embedded in the first one with a smaller amplitude. We also examine the small-scale oscillations at $5-\mathrm{km}$ and $8-\mathrm{km}$ height, and find that oscillations at different heights exhibit the similar patterns with the same periods (figure not shown).

The simulated trochoidal oscillations is further compared with the observation. Figure $3 \mathrm{~b}$ shows the time series of the zonal and meridional track oscillations relative to the 3-h mean tracks of the simulated Rammasun during the period from 0230 UTC to 1030 UTC 18 July. The period is roughly the same with that in Fig. 3a. In agreement with the observation, the simulated track oscillation has a period of $\sim 4 \mathrm{~h}$. The simulated amplitude is only half of the observation during the period from 0230 UTC to 0700 UTC 18 
(a)

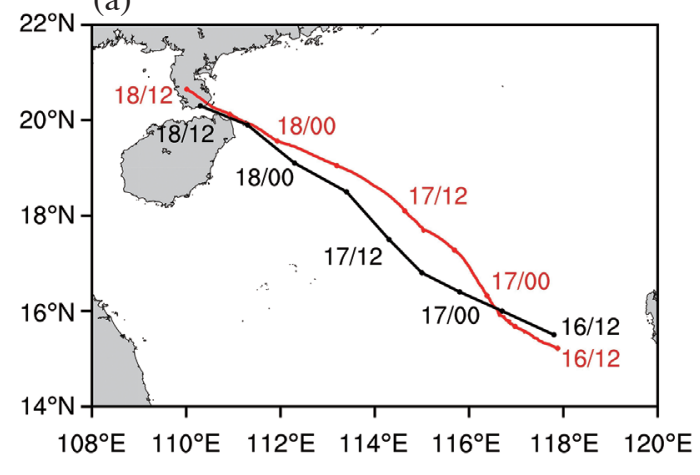

(b)

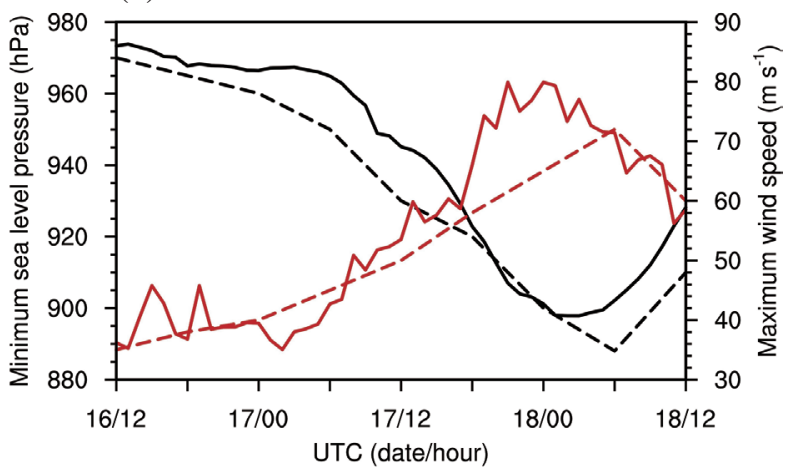

Fig. 6. (a) The observed (black) and simulated (red) tracks of Typhoon Rammasun (2014) during the period 1200 UTC 16 July to 1200 UTC 18 July. (b) The observed (dashed) and simulated (solid) maximum wind speed (red) and minimum sea level pressure (black) at 1-h intervals during the period 1200 UTC 16 July to 1200 UTC 18 July.
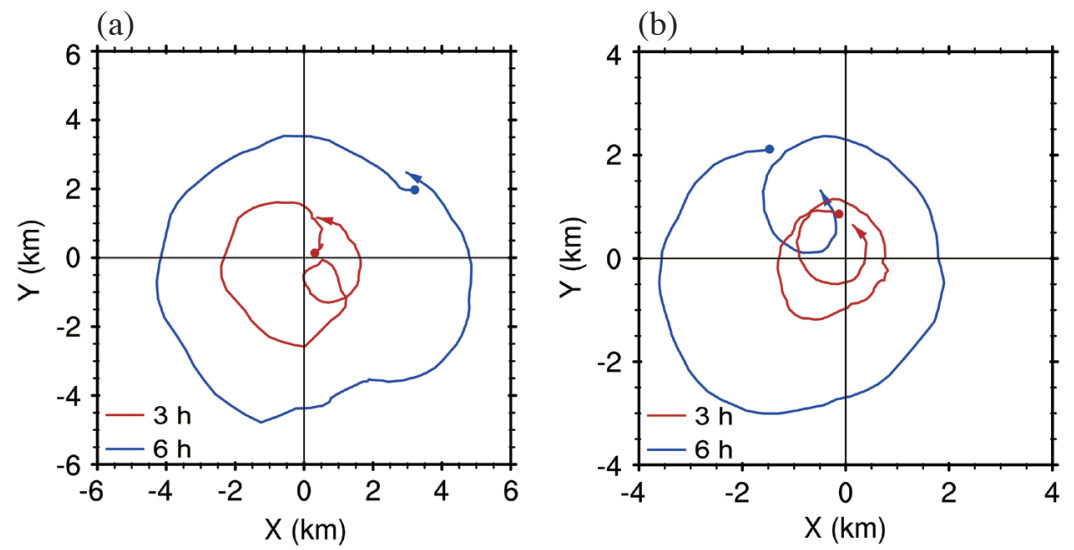

Fig. 7. The simulated cyclonic trajectory at 3-km height of the track oscillations (km) relative to the 3-h (red) and 6-h (blue) mean tracks of the simulated Rammasun during the period (a) 0600 UTC 17 July to 1400 UTC 17 July and (b) 2200 UTC 17 July to 0600 UTC 18 July. The rotation direction is indicated by arrows.

July, while it is very similar with the observation during the period from 0700 UTC to 1030 UTC 18 July. For the 6-h mean track, the period and amplitude of the simulated track oscillations are $\sim 4 \mathrm{~h}$ and $\sim 3 \mathrm{~km}$ during the period from 0230 UTC to 0900 UTC 18 July (figure not shown). The amplitude is also smaller than the observation. Nolan et al. (2001) found that the small-scale trochoidal oscillations is associated with the instability of the wave-number-one component of TC circulation. Wu and Chen (2016) found that the oscillations result from the asymmetric dynamics of the TC inner-core. The different amplitude between the simulation and observation suggests that the current simulation did not well capture TC inner-core dynamics.

To further demonstrate the oscillation of the inner core circulation, the trajectory of the track oscillations is also derived with the MTC method. Based on the MVC and MTC, Fig. 8 shows comparisons of the track oscillations relative to the 6-h running mean tracks, indicating that the two methods lead to the similar oscillating patterns with the same pe- riods. The MVC leads to a relatively smooth rotation, while the MTC contains many abrupt changes. This is because the selected pressure field for the MVC is relatively smooth while the selected wind field for the MTC is considerably discontinuous, especially near RMW. We can see that the oscillation of the MTC is very similar to that of the MVC during the period from 2200 UTC 17 July to 0600 UTC 18 July. This suggests that the trochoidal oscillation is the wobble of the whole inner-core circulation. Although there are some differences in the amplitude between the simulation and the radar-based observation, it is suggested that the observed track oscillation can be numerically simulated in terms of the short period and small amplitude.

\section{SUMMARY}

The TC track oscillation has been investigated for several decades while no agreement has been reached about the mechanisms for the small-amplitude trochoidal oscillation 
(a)

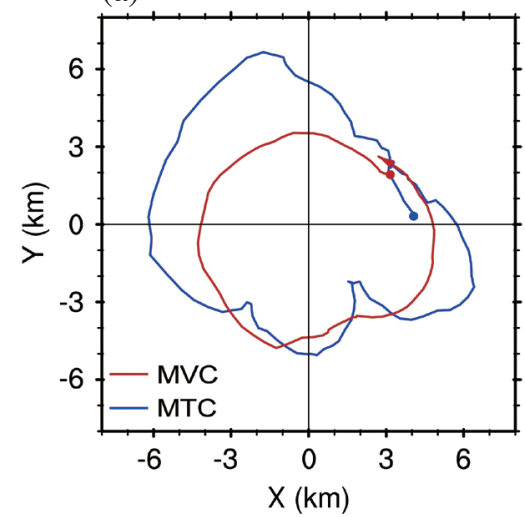

(b)

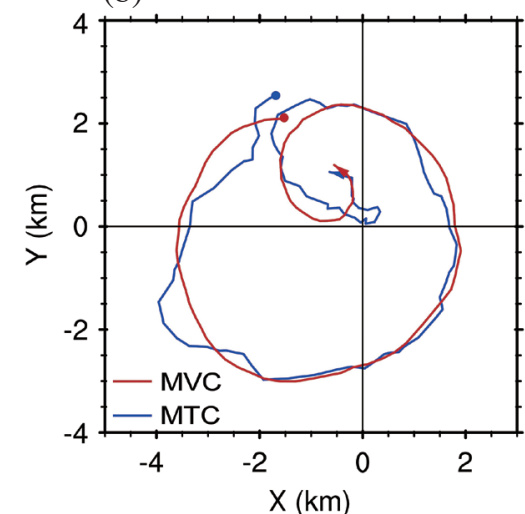

Fig. 8. The simulated cyclonic trajectory at 3-km height of the track oscillation $(\mathrm{km})$ relative to the 6-h mean tracks derived from the MVC (red) and MTC (blue) methods during the periods (a) from 0600 UTC 17 July to 1400 UTC 17 July and (b) from 2200 UTC 17 July to 0600 UTC 18 July. The rotation direction is indicated by arrows.

in TC motion. In this study, the small-amplitude trochoidal oscillation in the tracks of Super Typhoons Rammasun (2014) and Lekima (2019) is detected based on the 6-minute radar observation. The radar-based tracks of Typhoons Rammasun and Lekima exhibited small-amplitude track oscillations with the period of a few hours, which cannot be captured in the CMA real-time monitoring data with 0.1-degree resolution in latitude and longitude. In agreement with previous studies (Abe 1987; Hong and Chang 2005; Marks et al. 2008; Wu and Chen 2016; Yang et al. 2020), the trajectories of the observed trochoidal oscillations always exhibit cyclonic rotations.

The numerical simulation of Typhoon Rammasun was conducted to verify the detected track oscillation. It is indicated that the observed track oscillation can be numerically simulated in terms of the amplitude and period. The features of simulated oscillations derived from the MTC and the MVC were also similar to the radar-based observation in terms of the short period and small amplitude, indicating that the trochoidal oscillation is the wobble of the whole TC inner-core circulation. Since the small-scale track oscillations are closely associated with the dynamics of the TC inner-core, the trochoidal oscillation in TC tracks may provide important information for understanding the intensity and structure changes of TCs.

Acknowledgements This study was jointly supported by the National Natural Science Foundation of China (41730961, 42150710531, 41675051, and 41905001).

\section{REFERENCES}

Abe, S., 1987: The looping motion and the asymmetry of tropical cyclone. J. Meteorol. Soc. Jpn., 65, 247-258, doi: 10.2151/jmsj1965.65.2_247. [Link]
Bao, X., L. Wu, S. Zhang, H. Yuan, and H. Wang, 2020: A comparison of convective raindrop size distributions in the eyewall and spiral rainbands of Typhoon Lekima (2019). Geophys. Res. Lett., 47, e2020GL090729, doi: 10.1029/2020GL090729. [Link]

Cai, Q. and Y. Xu, 2016: An analysis of Super typhoon Rammasun's(2014) peak intensity. Geophysical Research Abstracts, Vol. 18, EGU2016-13654, EGU General Assembly 2016, Vienna, Austria.

Chang, P.-L., B. J.-D. Jou, and J. Zhang, 2009: An algorithm for tracking eyes of tropical cyclones. Weather Forecast., 24, 245-261, doi: 10.1175/2008waf2222112.1. [Link]

Dai, H., K. Zhao, Q. Li, W. Lee, J. Ming, A. Zhou, X. Fan, Z. Yang, F. Zheng, and Y. Duan, 2021: Quasiperiodic intensification of convective asymmetries in the outer eyewall of Typhoon Lekima (2019). Geophys. Res. Lett., 48, e2020GL091633, doi: 10.1029/2020GL091633. [Link]

Dudhia, J., 1989: Numerical study of convection observed during the winter monsoon experiment using a mesoscale two-dimensional model. J. Atmos. Sci., 46, 30773107, doi: 10.1175/1520-0469(1989)046<3077:NSOC OD>2.0.CO;2. [Link]

Feng, Y., L. Wu, Q. Liu, and W. Zhou, 2021: Negative Pressure Perturbations Associated with Tornadoscale Vortices in the Tropical Cyclone Boundary Layer. Geophys. Res. Lett., 48, e2020GL091339, doi: 10.1029/2020GL091339. [Link]

Holland, G. J. and M. Lander, 1993: The Meandering Nature of Tropical Cyclone Tracks. J. Atmos. Sci., 50, 12541266, doi: 10.1175/1520-0469(1993)050<1254:TMN OTC $>2.0 . \mathrm{CO} ; 2$. [Link]

Hong, J.-S. and P.-L. Chang, 2005: The trochoid-like track in Typhoon Dujuan (2003). Geophys. Res. Lett., 32, 
L16801, doi: 10.1029/2005GL023387. [Link]

Hong, S. Y. and J.-O. J. Lim, 2006: The WRF single-moment 6-class microphysics scheme (WSM6). Asia-Pac. J. Atmos. Sci., 42, 129-151.

Horn, J. D., 1951: On Irregular Movements of Tropical Cyclones in the Pacific. Bull. Amer. Meteorol. Soc., 32, 344-345, doi: 10.1175/1520-0477-32.9.344. [Link]

Itano, T., G. Naito, and M. Oda, 2002: Analysis of elliptical eye of Typhoon Herb (T9609). Sci. Eng. Rep. Natl. Def. Acad., 39, 9-17. (in Japanese with English abstract)

Jones, R. W., 1977: Vortex motion in a tropical cyclone model. J. Atmos. Sci., 34, 1518-1527, doi: 10.1175/1520-0469(1977)034<1518:VMIATC >2.0. CO;2. [Link]

Jordan, C. L., 1966: Surface pressure variations at coastal stations during the period of irregular motion of Hurricane Carla of 1961. Mon. Weather Rev., 94, 454-458, doi: 10.1175/1520-0493(1966)094<0454:SPVACS>2 . 3.CO;2. [Link]

Jordan, H. M. and D. J. Stowell, 1955: Some small-scale features of the track of Hurricane Ione. Mon. Weather Rev., 83, 210-215, doi: 10.1175/1520-0493(1955)083<0210:SSFOTT>2.0. $\mathrm{CO} ; 2$. [Link]

Kain, J. S. and J. M. Fritsch, 1993: Convective parameterization for mesoscale models: The Kain-Fritsch scheme. In: Emanuel, K. A. and D. J. Raymond (Eds.), The Representation of Cumulus Convection in Numerical Models, Meteorological Monographs, American Meteorological Society, Boston, MA, 165-170, doi: 10.1007/978-1-935704-13-3_16. [Link]

Khandekar, M. L. and G. V. Rao, 1971: The Mutual Interaction of Multiple Vortexes and its Influence on Binary and Single Tropical Vortex Systems. Mon. Weather Rev., 99, 840-846, doi: 10.1175/1520-0493(1971)099<0840:TMIOMV>2.3. $\mathrm{CO} ; 2$. [Link]

Kuo, H. L., 1969: Motions of vortices and circulating cylinder in shear flow with friction. J. Atmos. Sci., 26, 390398, doi: 10.1175/1520-0469(1969)026<0390:MOVA $\mathrm{CC}>2.0 . \mathrm{CO} ; 2$. [Link]

Lawrence, M. B. and B. M. Mayfield, 1977: Satellite observations of trochoidal motion during Hurricane Belle 1976. Mon. Weather Rev., 105, 1458-1461, doi: 10.1175/1520-0493(1977)105<1458:SOOTMD>2.0. CO;2. [Link]

Lu, X., H. Yu, M. Ying, B. Zhao, S. Zhang, L. Lin, L. Bai, and R. Wan, 2021: Western North Pacific tropical cyclone database created by the China Meteorological Administration. Adv. Atmos. Sci., 38, 690-699, doi: 10.1007/s00376-020-0211-7. [Link]

Marks, F. D., P. G. Black, M. T. Montgomery, and R. W. Burpee, 2008: Structure of the Eye and Eyewall of
Hurricane Hugo (1989). Mon. Weather Rev., 136, 1237-1259, doi: 10.1175/2007mwr2073.1. [Link]

Menelaou, K., M. K. Yau, and T.-K. Lai, 2018: A Possible Three-Dimensional Mechanism for Oscillating Wobbles in Tropical Cyclone-Like Vortices with Concentric Eyewalls. J. Atmos. Sci., 75, 2157-2174, doi: 10.1175/JAS-D-18-0005.1. [Link]

Mirocha, J. D., J. K. Lundquist, and B. Kosović, 2010: Implementation of a Nonlinear Subfilter Turbulence Stress Model for Large-Eddy Simulation in the Advanced Research WRF Model. Mon. Weather Rev., 138, 4212-4228, doi: 10.1175/2010mwr3286.1. [Link]

Mlawer, E. J., S. J. Taubman, P. D. Brown, M. J. Iacono, and S. A. Clough, 1997: Radiative transfer for inhomogeneous atmospheres: RRTM, a validated correlated-k model for the longwave. J. Geophys. Res., 102, 16663 16682, doi: 10.1029/97jd00237. [Link]

Muramatsu, T., 1986: Trochoidal motion of the eye of Typhoon 8019. J. Meteorol. Soc. Jpn., 64, 259-272, doi: 10.2151/jmsj1965.64.2_259. [Link]

Noh, Y., W. G. Cheon, S. Y. Hong, and S. Raasch, 2003: Improvement of the K-profile model for the planetary boundary layer based on large eddy simulation data. Bound.-Layer Meteor., 107, 401-427, doi: 10.1023/a:1022146015946. [Link]

Nolan, D. S., M. T. Montgomery, and L. D. Grasso, 2001: The wavenumber-one instability and trochoidal motion of hurricane-like vortices. J. Atmos. Sci., 58, 32433270, doi: 10.1175/1520-0469(2001)058<3243:TWOI AT>2.0.CO;2. [Link]

Oda, M., M. Nakanishi, and G. Naito, 2006: Interaction of an asymmetric double vortex and trochoidal motion of a tropical cyclone with the concentric eyewall structure. J. Atmos. Sci., 63, 1069-1081, doi: 10.1175/ JAS3670.1. [Link]

Qiu, W., L. Wu, and F. Ren, 2020: Monsoonal Influences on Offshore Rapid Intensification of Landfalling Typhoons in a Sheared Environment over the South China Sea. Weather Forecast., 35, 623-634, doi: 10.1175/ waf-d-19-0134.1. [Link]

Reasor,P.D. and M.T.Montgomery,2001:Three-dimensional alignment and corotation of weak, TC-like vortices via linear vortex Rossby waves. J. Atmos. Sci., 58, 23062330, doi: 10.1175/1520-0469(2001)058<2306:TDA $\mathrm{ACO}>2.0 . \mathrm{CO} ; 2$. [Link]

Willoughby, H. E., 1988: Linear motion of a shallow-water, barotropic vortex. J. Atmos. Sci., 45, 1906-1928, doi: 10.1175/1520-0469(1988)045<1906:LMOASW>2.0 . CO;2. [Link]

Wu, L. and X. Chen, 2016: Revisiting the steering principal of tropical cyclone motion in a numerical experiment. Atmos. Chem. Phys., 16, 14925-14936, doi: 10.5194/ acp-16-14925-2016. [Link]

Yang, H., L. Wu, and T. Xie, 2020: Comparisons of four 
methods for tropical cyclone center detection in a highresolution simulation. J. Meteorol. Soc. Jpn., 98, 379393, doi: 10.2151/jmsj.2020-020. [Link]

Yeh, T. C., 1950: The motion of tropical storms under the influence of a superimposed southerly current. J. Atmos. Sci., 7, 108-113, doi: 10.1175/1520-0469
(1950)007<0108:TMOTSU>2.0.CO;2. [Link]

Ying, M., W. Zhang, H. Yu, X. Lu, J. Feng, Y. Fan, Y. Zhu, and D. Chen, 2014: An overview of the China Meteorological Administration tropical cyclone database. $J$. Atmos. Ocean. Technol., 31, 287-301, doi: 10.1175/ jtech-d-12-00119.1. [Link] 